Article

\title{
Optimization and Comparative Analysis of Traffic Restriction Policy by Jointly Considering Carpool Exemptions
}

\author{
Xueyan Wei ${ }^{1,2,3, *}$, Weijie Yu ${ }^{1,2,3}$, Wei Wang ${ }^{1,2,3, *}$, De Zhao ${ }^{1,2,3}$ and Xuedong Hua ${ }^{1,2,3}$ \\ 1 Jiangsu Key Laboratory of Urban ITS, Southeast University, No.2 Southeast University Road, Nanjing \\ 211189, China; 213153324@seu.edu.cn (W.Y.); zhaode_0@aliyun.com (D.Z.); 101012566@seu.edu.cn (X.H.) \\ 2 Jiangsu Province Collaborative Innovation Center of Modern Urban Traffic Technologies, Southeast \\ University, Nanjing 211189, China \\ 3 School of Transportation, Southeast University, Nanjing 211189, China \\ * Correspondence: weixy163@163.com (X.W.); wangwei_transtar@163.com (W.W.)
}

Received: 20 August 2020; Accepted: 16 September 2020; Published: 18 September 2020

\begin{abstract}
As a countermeasure to urban exhaust pollution and traffic congestion, traffic restriction policy (TRP) and carpooling strategy have been widely introduced throughout the world. However, their effects are largely determined by the rationality of implementing policies, and unreasonable policies make them controversial on the long-term implementation benefits. To more effectively manage traffic demand and maintain the sustainability of transportation system, it is necessary to make optimization for management policy before implementation. In this paper, the elastic demand model and equilibrium assignment model are developed under TRP. Considering the negative impact of the mandatory TRP on the public acceptance, we propose a novel TRP strategy, namely TRP with carpool exemptions (TRP-CE), that is, a proportion of high occupancy vehicles (HOV) are allowed to travel in the restricted district even if their license plate numbers are restricted. Then, a bi-level programming model is proposed to solve the optimal schemes by combining multi purposes of ensuring travel convenience, alleviating traffic congestion, and reducing the exhaust pollution. Finally, a numerical experiment is conducted to evaluate the effectiveness of proposed models and make comparative analysis between separate TRP and TRP-CE. The results indicate that TRP-CE has benefits in the following aspects: (1) Carpool exemptions provide an incentive to carpool for travelers by private cars; (2) the public acceptance of TRP is improved by introducing carpool exemptions as a compensatory mitigation strategy for mandatory TRP; (3) the implementation effect of demand management can be well achieved by joint optimization; and (4) there is no need to design and reconstruct HOV lanes for the implementation of TRP-CE, which is convenient for practical application.
\end{abstract}

Keywords: traffic restriction; traffic demand management; carpooling; traffic policy; bi-level programming model

\section{Introduction}

As a traffic demand management (TDM) strategy, traffic restriction policy (TRP) is proposed to alleviate traffic congestion by controlling the number of vehicles on the network. TRP is firstly introduced in the form of odd-and-even license plate rule in 2007, aiming to manage traffic demand and reduce exhaust pollution in Beijing. Recent years, traffic demand has been gradually approaching or even exceeding traffic supply with the explosive growth of private cars in many cities around the world. TRP has been considered as one of the most directed measures to alleviate traffic congestion and 
exhaust pollution. For better implementation benefits, effectiveness evaluation and policy optimization are essential before implementation of TRP.

However, effective TDM measures are difficult to design and implement. Apart from technological and legal problems, the lack of public support is usually recognized as one of main obstacles preventing TDM measures from achieving their expected goals. Public acceptance could affect whether a TDM policy will effectively modify travelers' behaviors, and thus determine the effect of the policy in future [1,2]. To better understand the commuters' acceptance of TRP, a survey has been conducted with respondents sampled from car-owners commuting within a restricted area of Tianjin in China [3]. Based on the survey data, the researchers conclude that the benefits perceived by commuters, such as the improvement in traffic congestions, is a priority for earning the public acceptance of TRP. Furthermore, they point out that the policies should not be overly rigid, otherwise commuters will feel more negative effects (i.e., a decrease in the level of "perceived cost-benefit"), which may then lower the level of acceptance.

For improving the public acceptance of TRP and guiding traffic demand shifting from private cars to public transits, reasonable optimizations on restricted proportion and restricted district should be designed; besides, supporting measures such as improving the service level of alternative modes and restricting the purchase of private cars should be implemented together with TRP [3-6]. Researchers [7-9] propose that TRP has great potential to replace or supplement the road tolling policy and is possible to realize Pareto improvement of resource allocation in transportation system through policy optimization. To understand the optimization of TRP, Shi and Xu et al. (2014) [10,11] construct a bi-level programming model to reflect the Stackelberg game between policy makers and travelers, and further solve the network equilibrium with elastic demand under long-term TRP. The results show that the bi-level programing model can well deal with the optimization problem of TRP under variable traffic demand and road capacity. Furthermore, Nie (2017) [12] establishes a mathematical model considering the travelers' heterogeneity and mode-choice behaviors, and illustrates that it is possible that no matter how the TRP is designed, the system cost cannot be reduced.

Similar to TRP, carpooling originated in the United Stated and is also one of the most common demand management strategies. By setting up the high occupancy vehicle (HOV) lanes, private car travelers are encouraged to carpool to fully utilize the limited traffic resources. Recently, carpooling is encouraged in cities of Asia and Europe, and HOV lanes are set up in Shenzhen, Chengdu, Jinan, and so on in China. As both TRP and carpooling strategy belong to demand management strategy, it is possible to combine the TRP with carpooling strategy to achieve complementary advantages. Some researchers study the joint optimization problem of TRP and HOV lane setting, for instance, Ding (2018) [13] optimizes the network equilibrium assignment under TRP and makes comparative analysis between situations of whether setting HOV lanes or not. Regarding this, the travel cost model and equilibrium assignment model are proposed by comprehensively considering carpooling, TRP, and speed limit, then the bi-level programming model is developed to solve the optimal restricted district and restricted proportion.

Learning from the existing research on joint optimization of TRP and carpool strategy, some limitations still exist: (1) There is insufficient analysis on the route detour and temporary mode shifting caused by TRP when developing the equilibrium assignment model; (2) TRP affects the travel intention to some extent, further induces the elastic change of traffic demand, and meanwhile, the shares of travel modes change complying with rules of TRP, of which conjoint analysis is ignored in the existing research; (3) previous optimization of TRP and carpooling strategy merely concentrates on a single implementation purpose, namely easing traffic congestion or reducing exhaust pollution. In reality, both TRP and carpooling strategy are always determined by decision makers with multiple purposes based on weight coefficients. Furthermore, the implementation effect of carpooling strategy is largely determined by the layout of HOV lanes. Unreasonable HOV-lane design and unmotivated willingness to carpooling make the implementation effect of usual carpooling strategy controversial, so that further optimization is necessary. 
From the above analysis, this study proposes a novel TRP strategy by jointly considering carpool exemptions (TRP-CE). Based on the original TRP rules, proportions of HOVs are allowed to travel in the restricted districts even if their license plate numbers are restricted. By comparative analysis between separate TRP and TRP-CE, TRP-CE provides an incentive to carpool for travelers by private cars together with a mild TRP, so that the public acceptance of TRP would be improved. Furthermore, the implementation effect of demand management strategy can be ensured by joint optimization. Due to carpool exemptions, restricted carpooling cars and unrestricted private cars have the same right of way in restricted districts. Therefore, there is no need to design and reconstruct HOV lanes for the implementation of TRP-CE, which is convenient for practical application.

The remainder of this study is organized as follows. In Section 2, the elastic demand and generalized travel cost are analyzed under TRP. Section 3 proposes the bi-level programming model for TRP optimization. In Section 4, TRP-CE is introduced, and the joint optimization model is proposed with comprehensive analysis of traffic demand and travel cost. Section 5 designs a numerical experiment and makes a comparison between TRP and TRP-CE, and further conducts sensitivity analysis of carpool cost. Section 6 concludes the study with a summary of main findings and directions for future research.

\section{Analysis on Elastic Demand and Generalized Travel Cost under TRP}

Elastic demand should be considered when analyzing the long-term effect of traffic restriction on network equilibrium. The relationship between actual traffic demand and potential maximum demand meets:

$$
Q_{w}=\tilde{Q}_{w} \exp \left(-\eta \Phi_{w}\right), \forall w
$$

where $\eta$ is the elasticity coefficient and $\Phi_{w}$ is the expected minimum generalized cost of OD $w$. Based on the discrete choice theory [14], the expected minimum travel cost is a function of the log-sum average cost of all mode $m \in M[10,15]$ and can be calculated as follows:

$$
\Phi_{w}=-\frac{1}{\theta} \ln \sum_{m \in M} \exp \left(-\theta \Phi_{w}^{m}\right), \forall w
$$

where $\theta$ is a parameter related to the variance of travelers' mode choice behavior, $\Phi_{w}^{m}$ denotes the expected minimum estimated travel cost of OD pair $w$ by mode $m$. Then, the actual traffic demand of mode $m$ can be calculated with Logit-based discrete selection model, given as:

$$
\frac{Q_{w}^{m}}{Q_{w}}=\frac{\exp \left(-\theta \Phi_{w}^{m}\right)}{\sum_{k \in M} \exp \left(-\theta \Phi_{w}^{k}\right)}, \forall w, m
$$

For the existing problems that ignore route detour and temporary mode shifting caused by TRP when developing the equilibrium assignment model, Wei et al. [16] propose the concept of mode shifting rate under TRP, based on which the changed traffic demand structure is calculated. We follow this analyzing method and consider travel modes of three categories, namely private car, taxi, and bus, denoted as $m \in M=\{c, r, b\}$. Then, classify the traffic demand under TRP into six categories: (1) Unrestricted traffic demand of private car, (2) restricted traffic demand of private car, which is completed with detour, (3) original traffic demand of taxi, (4) restricted traffic demand of private car shifting to taxi as the alternative mode, (5) original traffic demand of bus, and (6) restricted traffic demand of private car shifting to bus as the alternative mode. Travel type corresponding to six categories of traffic demand is denoted as $i \in I=\{c, c c, r, r c, b, b c\}$ in sequence. It should be noted that travelers in travel types $i \in\{c, c c, r c, b c\}$ actually own a private car, so that they belong to travel mode $m=c$.

To calculate the generalized trip cost of path $p \in P_{w}^{i}$ by travel type $i$ between OD $w$, we introduce $V_{w}^{p l i}$ as the stable travel consumption of path $p$, jointly determined by path $p$ and travel type $i$ between 
OD $w$, and $V_{w}^{i}$ as the stable travel consumption, path-independent and determined by travel type $i$. Similar to previous authors [17], we employ $v^{m}, \alpha^{m}$, and $\beta^{m}$ as the value of time, a unit using cost and extra travel consumption for mode $m$, respectively, and $t_{p}^{i}$ as the travel time of path $p$ by travel type $i$. Consequently, travel consumptions of various travel types under TRP are given as:

$$
\begin{gathered}
V_{w}^{p l i}=\left(v^{i}+\alpha^{i}\right) t_{p}^{i}, \forall i \in I, w \in W, p \in P_{w}^{i} \\
V_{w}^{i}= \begin{cases}\beta^{c}, & i \in\{c, c c\} \\
\beta^{r} v^{r}, & i=r \\
\beta^{r} v^{c}+\beta^{c}, & i=r c \\
\beta^{b} v^{b}, & i=b \\
\beta^{b} v^{c}+\beta^{c}, & i=b c\end{cases}
\end{gathered}
$$

Based above analysis, the $\Phi_{w}^{m}$ in Equations (1) and (3) are established as:

$$
\left\{\begin{array}{l}
\Phi_{w}^{c}=\sum_{i \in\{c, c c, r c, b c\}} \rho_{w}^{i}\left(V_{w}^{i}+\bar{V}_{w}^{i}+\frac{1}{\theta} \ln \rho_{w}^{i}\right) \\
\Phi_{w}^{r}=V_{w}^{r}+\bar{V}_{w}^{r} \\
\Phi_{w}^{b}=V_{w}^{b}+\bar{V}_{w}^{b}
\end{array}\right.
$$

where $\rho_{w}^{i}$ is the percentage of travelers of travel type $i$ among all those of mode $m=c$, which is determined by TRP scheme based on mode shifting rate $\gamma_{w}$, and $\bar{V}_{w}^{i}$ is the excepted travel consumption of travel type $i$ between OD $w$. Additionally, the $\Phi_{w}^{i}$ for the calculation of $\gamma_{w}$ is obtained by:

$$
\Phi_{w}^{i}=V_{w}^{i}+\bar{V}_{w}^{i}, i \in\{c c, r c, b c\}
$$

\section{Optimization Model for TRP}

Combined with the practical application, this study considers three factors of travel convenience, traffic congestion, and exhaust pollution when making optimization of TRP. When TRP is adopted for alleviating exhaust pollution, the objective is set to minimize the total vehicle hours in the network while ensuring the traffic convenience. When TRP is adopted for easing traffic congestion, it is preferable to set the objective as minimizing the total overload flow of network while ensuring the traffic convenience, in which, traffic convenience is denoted by the travel intention $\frac{1}{\eta} \sum_{w \in W} Q_{w}$, the total vehicle hours in all links $l \in L$ are given by $\sum_{l \in L} x_{l} t_{l}^{c}\left(x_{l}\right)$, and overload flow is set to $\sum_{l \in L} \max \left\{0, x_{l}-c a p_{l}\right\}$, where $\mathrm{cap}_{l}$ denotes the capacity of link $l \in L$.

Furthermore, we introduce the positive coefficients $\omega_{1}$ and $\omega_{2}$ to represent the attention weights for the purpose of reducing air pollution and easing traffic congestion, respectively. Consequently, the upper-level programming model for optimization of $\operatorname{TRP}(\lambda, \tilde{L})$ is given as Equation (8), in which there are restricted proportion $\lambda \in[0,1]$ and restricted links $\tilde{L} \subset L$.

$$
\begin{aligned}
& \max _{(\lambda, \tilde{L})} Z=\frac{1}{\eta} \sum_{w \in W} Q_{w}-\omega_{1} \sum_{l \in L} x_{l} t_{l}^{c}\left(x_{l}\right)-\omega_{2} \sum_{l \in L} \max \left\{0, x_{l}-c a p_{l}\right\} \\
& \text { s.t. }\left\{\begin{array}{c}
0 \leq \lambda \leq 1 \\
\tilde{L} \subset L
\end{array}\right.
\end{aligned}
$$

where $Q_{w}, x_{l}$ are firstly solved by the lower-level equilibrium model under TRP scheme given by the upper-level model, then introduced into the upper-level model to calculate the target value of objective function. We introduce $\left(X^{*}, Q^{*}\right)$ to denote the equilibrium travel flow and demand structure of traffic 
network under TRP scheme $(\lambda, \tilde{L})$. According to the conservation of traffic demand and path flow, the feasible region of $(\boldsymbol{X}, \boldsymbol{Q})$ is established as:

$$
\Omega=\left\{\begin{array}{l}
\sum_{m \in M} Q_{w}^{m}=Q_{w}, \sum_{p \in P_{w}^{i}} f_{p}^{i}=q_{w}^{i}, \sum_{i \in I} q_{w}^{i}=Q_{w}, \\
\sum_{i \in I} \sum_{w \in W} \sum_{p \in P_{w}^{i}} \delta_{l p} f_{p}^{i}=x_{l}, f_{p}^{i} \geq 0, \forall w \in W, \forall i \in I, \forall p \in P_{w}^{i} ; \\
q_{w}^{i}=\rho_{w}^{i} Q_{w}^{c}, \forall i \in\{c, c c, r c, b c\}
\end{array}\right\}
$$

where $f_{p}^{i}$ is the flow on path $p \in P_{w}^{i}$ by travel type $i$ between OD $w ; q_{w}^{i}$ is the demand of travel type $i$ between OD $w ; \delta_{l p}$ is the element of the incidence matrix between links and paths, which equals " 1 " if link $l$ is on path $p$ and " 0 " otherwise.

Based on the demand function in Equations (1)-(3), the generalized travel cost (inverse demand) function for mode $m$ can be easily obtained, denoted as $\Phi_{w}^{m}(Q)$. The lower-level equilibrium model is then given by the bellow inequality model based on Corollary 1 in reference [17]:

$$
\sum_{w \in W} \sum_{i \in I} \sum_{p \in P_{w}^{i}}\left(V_{w}^{p \mid i}+V_{w}^{i}\right)\left(f_{p}^{i}-f_{p}^{i *}\right)+\sum_{w \in W} \sum_{m \in M} \Phi_{w}^{m}\left(Q^{*}\right)\left(Q_{w}^{m}-Q_{w}^{m *}\right) \geq 0, \forall(X, Q) \in \Omega
$$

The lower-level equilibrium assignment model is solved by TRP given by the upper-level model, then updated traffic demand and traffic flow are obtained and introduced to the upper-level model to calculate the target value. Learning from the existing research, Zhang et al. [18] adopt the Genetic Algorithm (GA) to solve the optimal restricted district and charging rate for TRP. Shi [10] and Ding [13] also develop GA to solve the optimal restricted district and restricted proportion, meanwhile verifying the effectiveness of GA with a specific case study. Consequently, this study follows them and develops GA based on graph theory to solve the bi-level programming model.

The gene coding of chromosome for TRP scheme $(\lambda, \tilde{L})$ includes two parts, respectively representing restricted proportion and restricted district. The fitness function of TRP scheme $\left(\lambda^{\prime}, \tilde{L}^{\prime}\right)$ is defined as Equation (11).

$$
F\left(\lambda^{\prime}, \tilde{L}^{\prime}\right)=Z\left(\lambda^{\prime}, \tilde{L}^{\prime}\right)-\min \{Z(\lambda, \tilde{L}) \mid(\lambda, \tilde{L}) \in \text { pop }\}
$$

where pop represents the contemporary population, and the individual fitness remains nonnegative. Note that if an invalid TRP scheme with unconnected restricted district and out-range restricted proportion is generated in the initial population or process of crossover and mutation, it must be discarded and another TRP scheme should be randomly generated until a valid TRP scheme is found.

\section{Joint Optimization of TRP Considering Carpool Exemptions}

This study proposes a novel traffic restriction policy considering carpool exemptions, that is, based on the original TRP rules, proportions of HOVs are allowed to travel in the restricted district even if their license plate numbers are restricted. The exempted HOVs are denoted as CEs. The key to joint optimization of TRP-CE is to determine the relationship between the restricted proportion of private cars and the exempted proportion of CEs.

\subsection{Analysis on Traffic Demand and Travel Cost for Joint Optimization}

The assumption that CEs carry two passengers while private cars only carry one passenger per trip is adopted in this study, which has been used and verified in the relative research on carpooling [13,19-22]. The exempted proportion of CEs is determined by traffic managers and issued to travelers in time through the modern Internet communication technology. The efficiency of carpooling is not considered in this study; that is, it is assumed that travelers can successfully carpool with other travelers with the same OD. However, additional travel costs are generated in the process of carpooling, such as additional time for seeking fellow passengers and the fee paid for carpooling. 
This study follows the existing assumptions that carpooling cost per trip is constant whether for drivers or passengers; that is, carpooling cost for drivers and passengers is the same and is denoted as $\beta^{s}$, in which " $s$ " is used to represent the relative variables of carpooling strategy. The alternative routes set for CEs is same with that for unrestricted private cars, which is denoted as $P_{w}^{c}$. Based on this, the generalized travel cost of CEs on route $p \in P_{w}^{c}$ are calculated as Equation (12).

$$
\left\{\begin{array}{l}
V_{w}^{p \mid s c}=\left(v^{c}+\alpha^{c}\right) t_{p}^{c} \\
V_{w}^{s c}=\beta^{s} \\
\Phi_{w}^{s c}=V_{w}^{p \mid s c}+V_{w}^{s c}
\end{array}\right.
$$

Traffic modes under TRP-CE include private car without carpool, CE, taxi, and bus in this study. When issuing restricted proportion $\lambda$ and exempted proportion for CEs $\psi$, trips by unrestricted private cars are not affected while those by restricted private cars will determine whether to change modes according to the detour rate. When the carpool exemption is sufficient, restricted travelers will choose the alternative mode among $\mathrm{CE}$, taxi, and bus according to their generalized travel cost. Otherwise, travelers can only choose alternative mode between taxi and bus. The mode changes of private cars under TRP-CE are shown in Figure 1a, in which the restricted district is filled with gray. For travelers whose neither trip destination nor origin is located in the restricted districts, denoted as $w \in O O$, there exist five travel types as illustrated in Figure 1b, while for those where at least one trip endpoint is restricted, denoted as $w \in I I \cup I O$, the travel type of detouring by restricted cars is not available and remaining travel types are illustrated in Figure 1c.

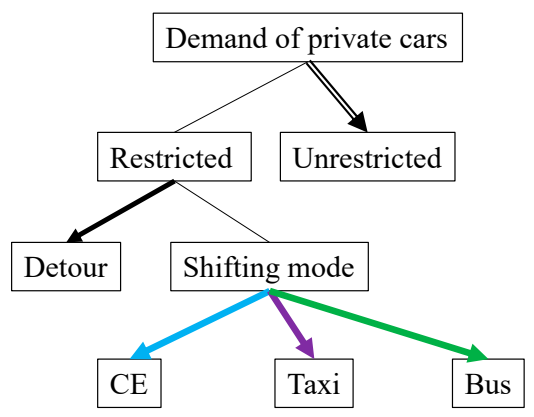

(a) Mode changes structure

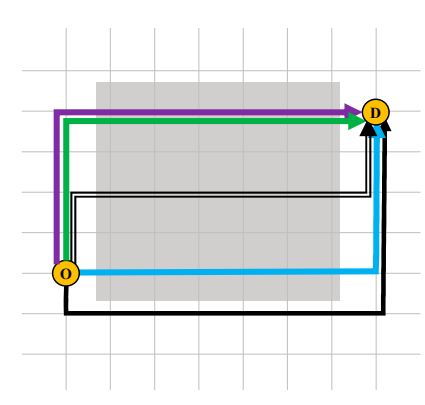

(b) $w \in O O$

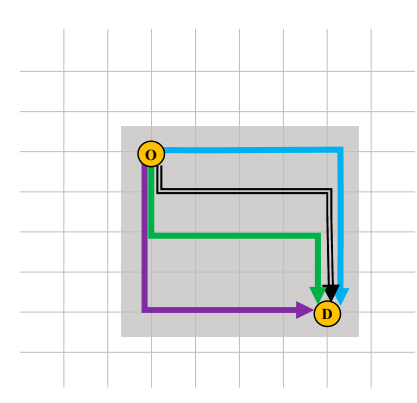

(c) $w \in I I \cup I O$

Figure 1. The mode changes of private cars under traffic restriction policy with carpool exemptions (TRP-CE).

Compared with the optimization of TRP, TRP-CE adds an alternative mode of CE. Consequently, mode shifting rate $\gamma_{w}$ for joint optimization is updated, as Equation (13).

$$
\gamma_{w}=\left\{\begin{array}{cc}
1.00 & w \in I I \cup I O \\
1.00-\frac{\exp \left(-\theta \Phi_{w}^{c c}\right)}{\sum_{i \in\{c,, r c, b c, s\}} \exp \left(-\theta \Phi_{w}^{i}\right)} & , w \in O O, \bar{\tau}_{w}^{c c} / \bar{\tau}_{w}^{c}>1.00 \\
0.00 & , w \in O O, \bar{\tau}_{w}^{c c} / \bar{\tau}_{w}^{c}=1.00
\end{array}\right.
$$

where $\bar{\tau}_{w}^{c c} / \bar{\tau}_{w}^{c}$ represents the detouring rate calculating by the shortest travel time of restricted and unrestricted private cars.

The ownership of private cars before TRP is denoted as $H_{w}^{c}$ and number of private car without carpool is set as $q_{w}^{0 c}=H_{w}^{c}$. When implementing TRP with restricted proportion $\lambda$, number of unrestricted private cars is $q_{w}^{c}=(1-\lambda) H_{w}^{c}$ and that of restricted private cars is $\lambda H_{w}^{c}$, in which number of restricted private cars choosing to detour is $\left(1-\gamma_{w}\right) \lambda H_{w}^{c}$ and that shifting to alternative modes is $\gamma_{w} \lambda H_{w}^{c}$. Based on the exempted proportion for CEs $\psi$, the maximum exempted number of CE in the network is $\psi \lambda H_{w}^{c}$, which can carry the maximum traffic demand $2 \psi \lambda H_{w}^{c}$. During the process of mode 
shift, the chosen probability of CE is calculated by Logit model with excepted estimated travel cost before TRP, as in Equation (14).

$$
p_{w}^{s c}=\min \left\{2 \psi, \frac{\gamma_{w} \exp \left(-\theta \Phi_{w}^{s c}\right)}{\sum_{i \in\{r c, b c, s c\}} \exp \left(-\theta \Phi_{w}^{i}\right)}\right\}
$$

Accordingly, the actual number of CE is $q_{w}^{s c}=\lambda q_{w}^{c} p_{w}^{s c} / 2$. Furthermore, the chosen probability of taxi $p_{w}^{r c}$ and that of bus $p_{w}^{b c}$ are calculated as Equation (15).

$$
\left\{\begin{array}{l}
p_{w}^{r c}=\frac{\left(\gamma_{w}-p_{w}^{s c}\right) \exp \left(-\theta \Phi_{w}^{r c}\right)}{\exp \left(-\theta \Phi_{w}^{r c}\right)+\exp \left(-\theta \Phi_{w}^{b c}\right)} \\
p_{w}^{b c}=\frac{\left(\gamma_{w}-p_{w}^{c c}\right) \exp \left(-\theta \Phi_{w}^{b c}\right)}{\exp \left(-\theta \Phi_{w}^{r c}\right)+\exp \left(-\theta \Phi_{w}^{b c}\right)}
\end{array}\right.
$$

Traffic demand shifting to taxi is calculated as $q_{w}^{r c}=p_{w}^{r c} \lambda H_{w}^{c}$ and that shifting to bus is calculated as $q_{w}^{b c}=p_{w}^{b c} \lambda H_{w}^{c}$. Finally, the quantitative relationship of mode changes under TRP-CE is summarized in Figure 2.

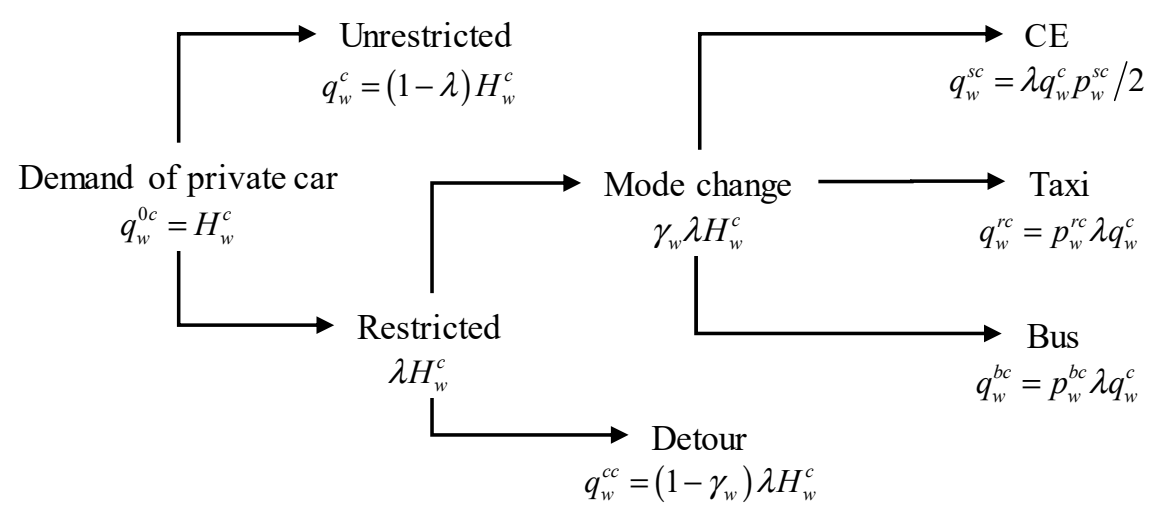

Figure 2. The quantitative relationship of mode changes under TRP-CE.

\subsection{Model of Joint Optimization for TRP-CE}

The joint optimization of TRP-CE can be considered as a Stackelberg Game between policy makers and travelers. When TRP scheme and carpooling strategy are designed, travelers respond to it by adjusting their travel behaviors. Furthermore, the decision makers adjust their decision again based on the target value of network equilibrium. This decision-making process can be realized, and the optimal TRP-CE scheme can be obtained by bi-level programming model.

As in Section 3, the upper-level programming model can be developed based on the TRP-CE $(\lambda, \tilde{L}, \psi)$, as Equation (16).

$$
\begin{aligned}
& \max _{(\lambda, \tilde{L}, \psi)} Z=\frac{1}{\eta} \sum_{w \in W} \sum_{i \in I} q_{w}^{i}+\beta^{s} \sum_{w \in W} q_{w}^{s c}-\omega_{1} \sum_{l \in L} x_{l} t_{l}^{c}\left(x_{l}\right)-\omega_{2} \sum_{l \in L} \max \left\{0, x_{l}-c a p_{l}\right\} \\
& \text { s.t. }\left\{\begin{aligned}
0 \leq \lambda \leq 1 \\
\tilde{L} \subset L \\
0 \leq \psi \leq 1
\end{aligned}\right.
\end{aligned}
$$

The lower-level programming model is the equilibrium assignment model under TRP-CE. By introducing additional alternative mode $\mathrm{CE}$, traffic demand and travel cost for joint optimization are calculated by referring to Section 4.2. Then equilibrium assignment is achieved using Equation (10). Learning from the existing research on TRP, GA based on graph theory is used to solve the bi-level programming model, in which the gene coding of TRP-CE includes restricted district $\tilde{L}$, restricted 
proportion $\lambda$, and exempted proportion $\psi$. The fitness function of TRP-CE $\left(\lambda^{\prime}, \tilde{L}^{\prime}, \psi^{\prime}\right)$ still remains nonnegative, as Equation (17).

$$
F\left(\lambda^{\prime}, \tilde{L}^{\prime}, \psi^{\prime}\right)=Z\left(\lambda^{\prime}, \tilde{L}^{\prime}, \psi^{\prime}\right)-\min \{Z(\lambda, \tilde{L}, \psi) \mid(\lambda, \tilde{L}, \psi) \in \operatorname{pop}\}
$$

\section{Numerical Experiments}

In this section, numerical experiments are presented to demonstrate the performance of proposed models, and comparative analysis between optimal schemes of TRP and TRP-CE are conducted simultaneously. All numerical experiments reported in this section are conducted on a Window 10 64-bit workstation with $3.2 \mathrm{GHz}$ Intel Core i7-8700 CPU and 16 GB RAM. All algorithms are coded and implemented in MATLAB.

\subsection{Basic Network and Multi Scenarios Design}

The typical network of Sioux Falls [23] is selected as the base network in numerical experiment. The network includes 24 nodes, 76 links, and 528 ODs, whose network topology is shown in Figure 3a. In this case, it is assumed that there is only one bus route for every OD, and travel time of bus is quadruple that of minimum travel time of private car under free-flow condition. Traffic demand given in Figure $3 \mathrm{~b}$ is set as the potential maximum traffic demand of private car before traffic restriction.

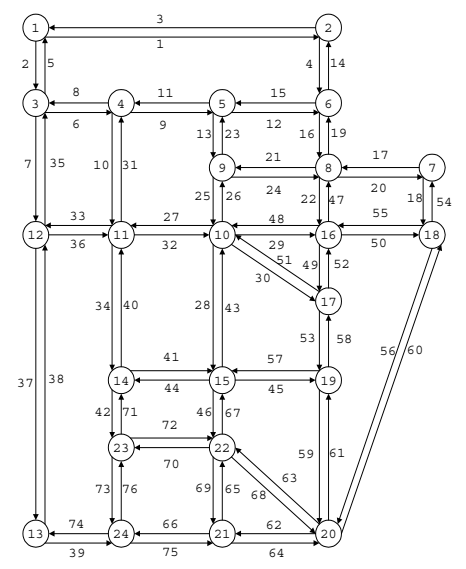

(a)

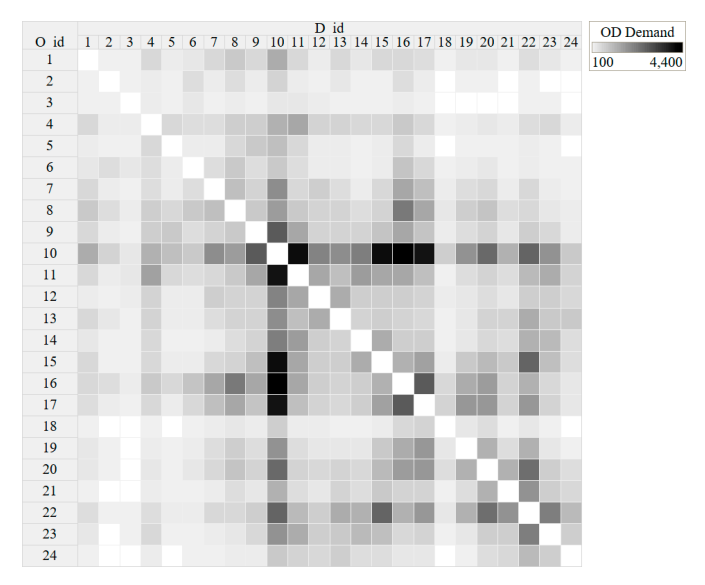

(b)

Figure 3. (a) Topology of base network; (b) initial traffic demand of base network.

Referring to reference [16], the parameters used in the numerical experiment are set as follows. The elastic coefficient $\eta$ is set to be 0.02 , while the sensitivity factor $\theta$ is set to be 1.00 . The unit using costs $\alpha^{c}, \alpha^{r}, \alpha^{b}$ are set to $0.4 \mathrm{RMB} / \mathrm{min}, 1.5 \mathrm{RMB} / \mathrm{min}$, and $0.1 \mathrm{RMB} / \mathrm{min}$, respectively. The extra cost of private car $\beta^{c}$ is set to $5 \mathrm{RMB} /$ trip. The extra times $\beta^{r}, \beta^{b}$ are set to $5 \mathrm{~min}$ and $10 \mathrm{~min}$, respectively. The carpool cost $\beta^{s}$ is $5 \mathrm{RMB} /$ trip. For GA algorithm, the crossing rate and variation rate are 0.90 and 0.01. The coefficients $\omega_{1}, \omega_{2}$ in the objective function of the upper-level model are both set to be 1.00 . Furthermore, the calculating function of travel time on links for car is given as Equation (18).

$$
t_{l}^{c}\left(x_{l}\right)=t_{l}^{0}\left[1.0+0.15\left(\frac{x_{l}}{c a p_{l}}\right)^{4}\right]
$$

where $t_{l}^{0}$ represents the travel time of car on link $l$ under free flow speed and cap ${ }_{l}$ represents the capacity of link $l$, whose values are obtained from the open-access website [23].

To solve the optimal TRP scheme and TRP-CE scheme in the Sioux Falls network, the following two scenarios are constructed and compared: 
S1: The theoretical optimal TRP scheme. By using the bi-level programming model shown in Equation (18), the optimal restricted district and restricted proportion are solved, in which the restricted district must be connected and the restricted proportion is reserved to percentile.

S2: The theoretical optimal TRP-CE scheme. By using the bi-level programming model shown in Equation (13), the optimal restricted district, restricted proportion, and exempted proportion are solved, in which the restricted district must be connected, and both restricted proportion and exempted proportion are reserved to percentile.

Furthermore, the base scenario without TRP or TRP-CE are also analyzed based on elastic demand and equilibrium assignment model. The base traffic flow and V/C distribution are shown in Figure 4 . Taking the base scenario as benchmark (BM), various traffic indicators of S1 and S2 are counted. The definition and formulas of indicators are summarized in Table 1.

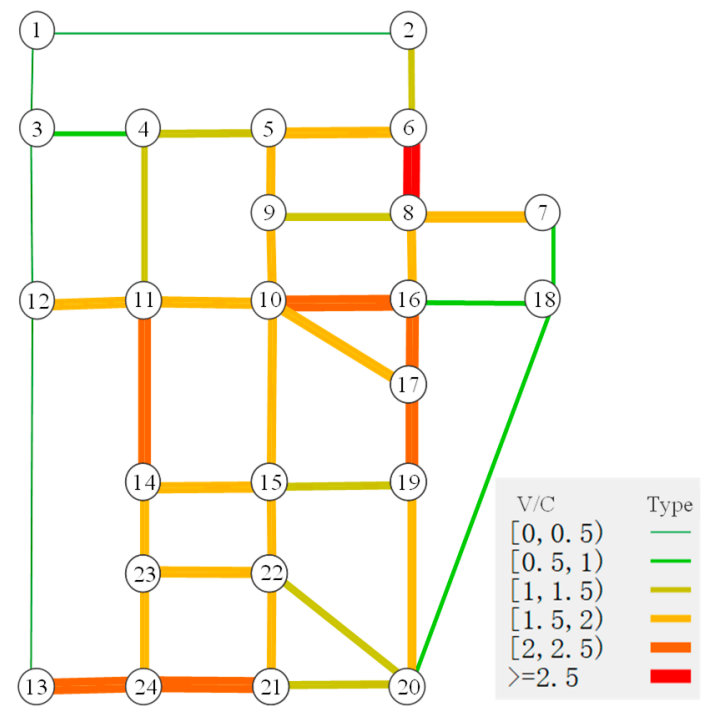

(a) $\mathrm{V} / \mathrm{C}$

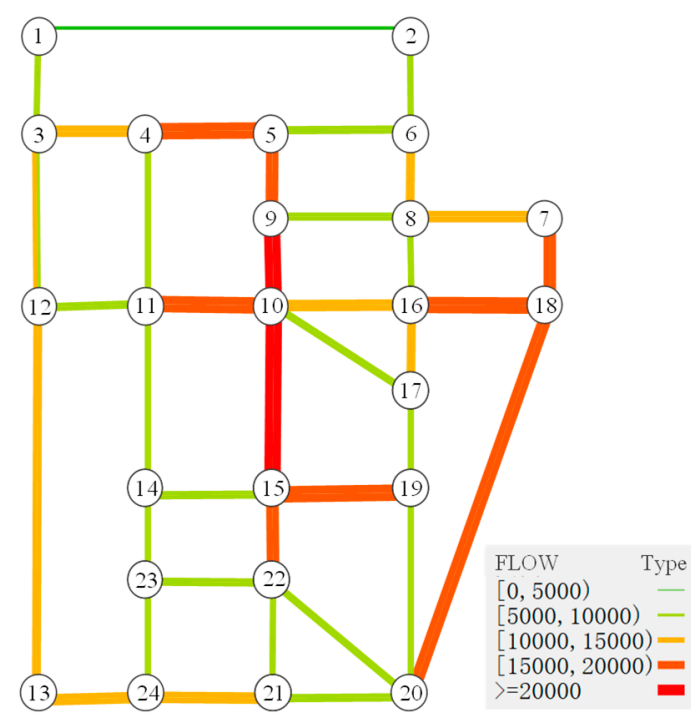

(b) flow distribution

Figure 4. (a) V/C of base scenario without TRP and TRP-CE; (b) flow distribution of base scenario without TRP and TRP-CE.

Table 1. Notations used for analysis of numerical experiment.

\begin{tabular}{ccc}
\hline Index & Definition & Formulas \\
\hline$S R^{m}$ & Share ratio of mode $m$. & $S R^{m}=\sum_{w \in W} q_{w}^{m} / \sum_{k \in M} \sum_{w \in W} q_{w}^{k}, m \in\{c, r, b\}$ \\
$Z$ & Target value of upper-level model. & Equations $(8)$ and $(13)$ \\
$T D$ & Total travel demand in studied network. & $T D=\sum_{w \in W} Q_{w}$ \\
$V H$ & Total vehicle hours. & $V H=\sum_{l \in L} \sum_{i \in I} x_{l}^{i} \cdot t_{l}^{i}, I=\{c, c c, r, r c\}$ \\
$O F$ & Total overload flow in studied network. & $O F=\sum_{l \in L} \max \left\{0, x_{l}-c a p_{l}\right\}$ \\
$T C$ & Travel willingness, as the indicator of travel convenience. & $T C=\frac{1}{\eta} T D=\frac{1}{\eta} \sum_{w \in W} Q_{w}$ \\
$H D^{s}$ & Total carpool cost in studied network. & $H D^{s}=\beta^{s} \sum_{w \in W}^{s c}$ \\
$O L$ & Number of overload links in the network. & $0 \leq O L \leq|L|$ \\
$A S$ & The average saturation of links. & $A S=A V G\left(x_{l} / c a p_{l}\right), \forall l \in L$ \\
$O S$ & The average overload saturation of links. & $O S=A V G\left(x_{l} / c a p_{l}\right), \forall l \in L_{O}$ \\
$M S$ & The maximal overload saturation of links. & $M S=M A X\left(x_{l} / c a p_{l}\right), \forall l \in L_{O}$ \\
\hline
\end{tabular}

\subsection{Analysis of Theoretical Optimal Schemes}

The theoretical optimal scheme of S1 and S2 are shown in Figure 5. From it, the restricted district of $\mathrm{S} 1$ is set around node 15 , involving 7 traffic nodes and 12 connected links, while that of S2 is set around node 10, involving 8 traffic nodes and 16 connected links. Furthermore, restricted proportion 
of $\mathrm{S} 1$ reaches $85 \%$, while that of S2 is only $76 \%$. For S2, there is an additional exempted proportion reaching $26 \%$.

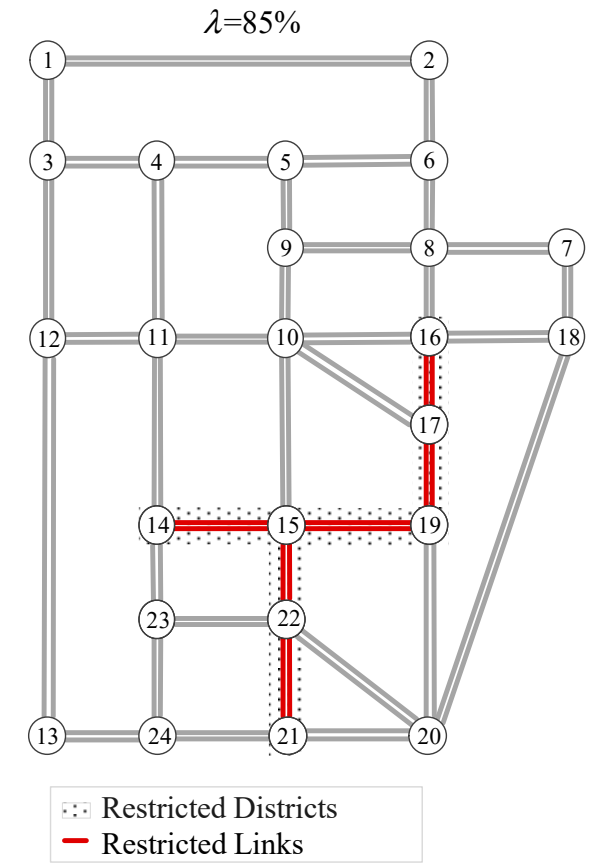

(a) S1

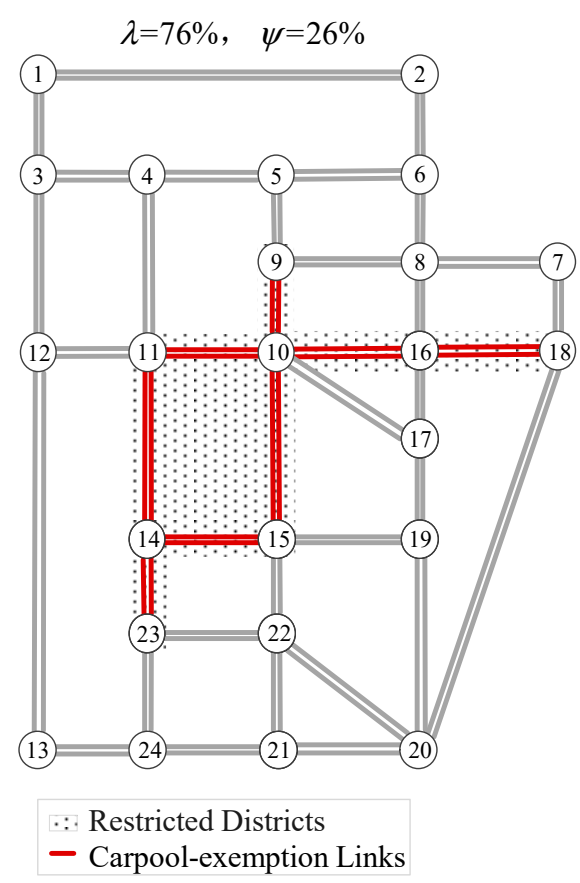

(b) S2

Figure 5. The theoretical optimal restricted district and restricted proportion of S1 and S2.

For further analyzing the effect of TRP and TRP-CE on V/C and flow distribution, the relative results are illustrated in Figure 6. Compared with the V/C in BM (Figure 4a), V/C of links in restricted district in S1 decreases as a whole, especially for links among nodes 15-19-17. Meanwhile, V/C of links near restricted district in $\mathrm{S} 1$ has also been improved compared with $\mathrm{BM}$, for instance, the traffic congestion of links including 6-8, 11-14, 10-16, and 24-21 has been effectively alleviated and low utilization of links including 1-3, 4-3, 3-12, and 12-13 has been effectively improved. Furthermore, the $\mathrm{V} / \mathrm{C}$ of links in the restricted district in $\mathrm{S} 2$ also decreases significantly compared with BM. Unlike $\mathrm{S} 1$, there is obvious congestion shifting caused by the large scope of restricted district, for instance, the traffic congestion of links including 19-20, 20-21, 15-22, and 22-23 is aggravated in S2.

Compared with the flow distribution of BM (Figure $4 \mathrm{~b}$ ), traffic flow of most links in the restricted district in S1 decreases and only four links among 19-15-22 have traffic flow more than 15,000. However, there are four links between 10-11 and 16-18 meeting "15,000 $\leq$ flow $<20,000$ " and four links among 9-10-15 meeting "flow $\geq 20,000$ " in S2. This phenomenon shows that more vehicles are affected in S2 than S1.

Various indicators of network equilibrium for BM, S1, and S2 are summarized in Table 2, in which $\mathrm{OL}$ in $\mathrm{BM}, \mathrm{S} 1$, and $\mathrm{S} 2$ is 60,54 , and 48 , respectively, indicating that $\mathrm{S} 1$ and $\mathrm{S} 2$ solve the overloading problems of 6 links and 12 links,. AS reflects the average service level of network. Obviously, both S1 and S2 have good effects, and S2 is more effective. For links still with an overloading problem, S1 has improvement on MS compared with BM while the congestion shift of S2 make MS worse. Furthermore, S2 is still the best in terms of OS.

From the indicators shown in Table 2, the target value $\mathrm{Z}$ of $\mathrm{S} 1$ is larger than that of BM. Meanwhile, S1 improves TD while reducing VH and OF compared with $\mathrm{BM}$, indicating that $\mathrm{S} 1$ maximizes the TRP effect while ensuring the travel convenience. For S2, the target value $Z$ minus total carpool cost $H D^{s}$ is equal to 25,221,028, which is larger than $Z$ in $S 1$. Furthermore, the travel intention $T D$ in $S 2$ is larger than that in S1, but total vehicle hours $V H$ and total overloading flow $O F$ in $\mathrm{S} 2$ are less than those in 
S1. The above four indicators indicate that the network equilibrium of S2 is better than that of S1. When implementing TRP, it is necessary to weigh in the feedback process of loss of travel convenience, improvement of traffic condition, and stimulation of travel intention. The proposed algorithm solving optimal TRP-CE scheme based on the implementation purpose can well meet the above requirements.

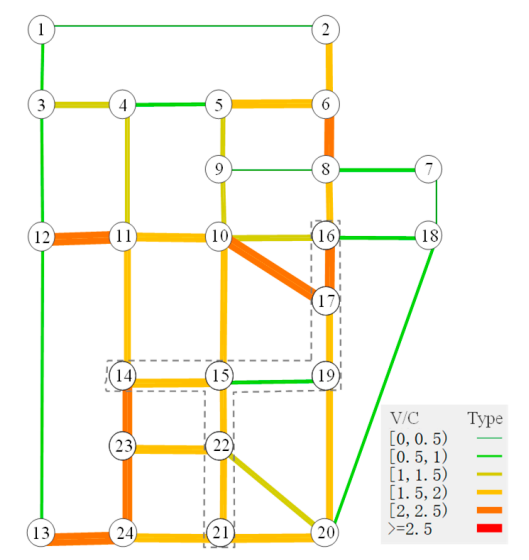

(a) $\mathrm{V} / \mathrm{C}$ distribution of $\mathrm{S} 1$

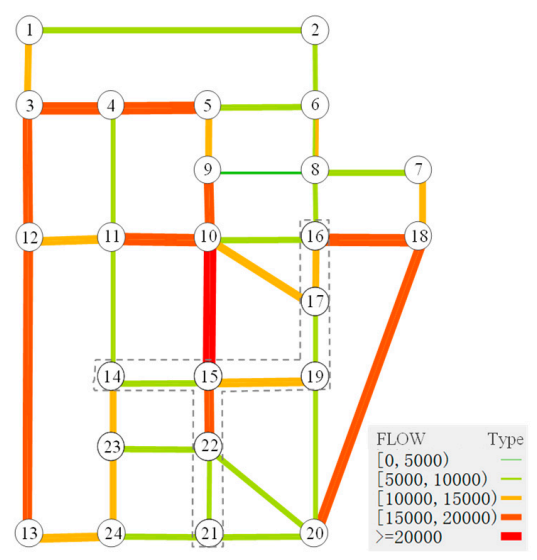

(c) Flow distribution of S1

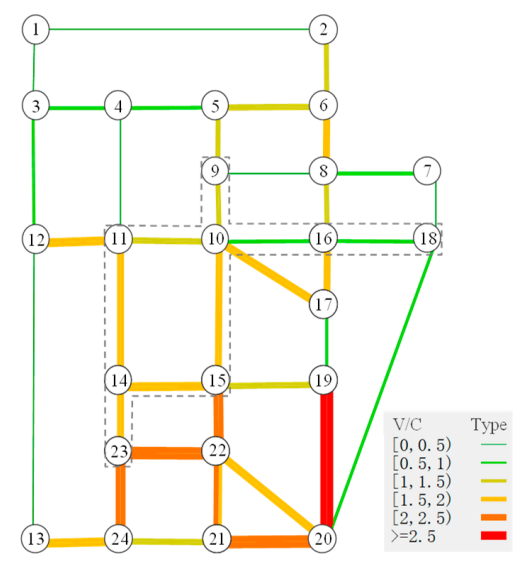

(b) V/C distribution of S2

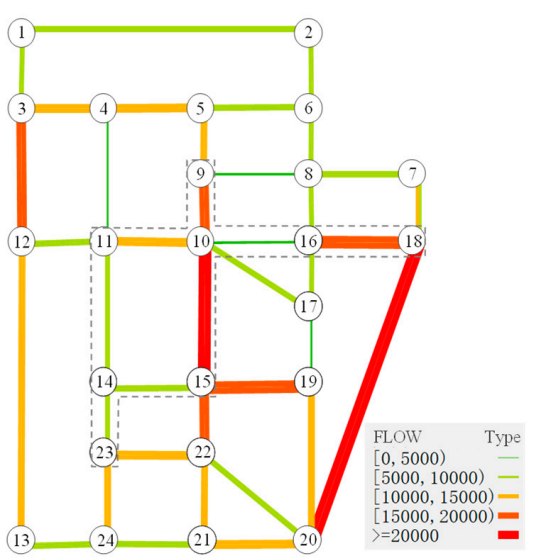

(d) Flow distribution of S2

Figure 6. Distribution of V/C and flow for S1 and S2.

Table 2. Comparison results among optimal restricted schemes under diversified scenarios.

\begin{tabular}{cccc}
\hline Index & BM & S1 & S2 \\
\hline$\lambda$ & - & $85 \%$ & $76 \%$ \\
$\psi$ & - & - & $26 \%$ \\
$O L$ & 60 & 54 & 48 \\
$A S$ & 1.75 & 1.42 & 1.28 \\
$O S$ & 1.96 & 1.71 & 1.68 \\
$M S$ & 2.65 & 2.32 & 2.79 \\
$Z$ & $23,123,734$ & $24,369,624$ & $25,514,499$ \\
$T D$ & 473,989 & 494,232 & 513,696 \\
$V H$ & $196,454.82$ & $121,897.12$ & $119,465.43$ \\
$O F$ & 379,271 & 220,058 & 197,587 \\
$S R^{c}$ & 0.36 & 0.17 & 0.25 \\
$S R^{r}$ & 0.21 & 0.38 & 0.36 \\
$S R^{b}$ & 0.43 & 0.45 & 0.39 \\
$H D^{s}$ & - & - & $293,471.59$ \\
\hline
\end{tabular}

From the perspective of demand structure under equilibrium network, the sharing rate of private car in S1 decreases significantly, but that of taxi and bus increases. Furthermore, the sharing rate of 
taxi increases more significantly compared with bus. It indicates that the taxi is more attractive as an alternative mode, since the generalized travel cost of bus is usually more than that of taxi due to the longer travel time and high value of time for restricted private car travelers. This result is also consistent with the survey result in the existing research [24]. Sharing rate of private car $S R^{c}$ in S2 is between that of S1 and BM. It can be explained that the restricted proportion $\lambda$ in S2 is less than S1 and S2 has additional exempted proportion $\psi$, which further leads to a lower decreased degree for sharing rate of private car. More significantly, the high occupancy of CEs ensures that the total vehicle hours in S2 are less than S1. The results further verify that TRP-CE can not only improve the travel convenience, but also optimize the network demand structure and enhance the beneficial effect on alleviating exhaust pollution and traffic congestion.

\subsection{Sensitivity Analysis on Carpool Cost}

To analyze the sensitivity of target value on carpool cost in the joint optimization model of TRP-CE, three common restricted proportions of $10 \%, 20 \%$, and $50 \%$, which correspond to restricting one or two digits of the license plate and the odd-and-even license plate rule, are tested with fixed restricted district and exempted proportion in S2. The sensitivity of target value to carpool cost under various restricted proportions is shown in Figure 7a, and the volatility of target value compared with mean value under various restricted proportions is shown in Figure 7c.

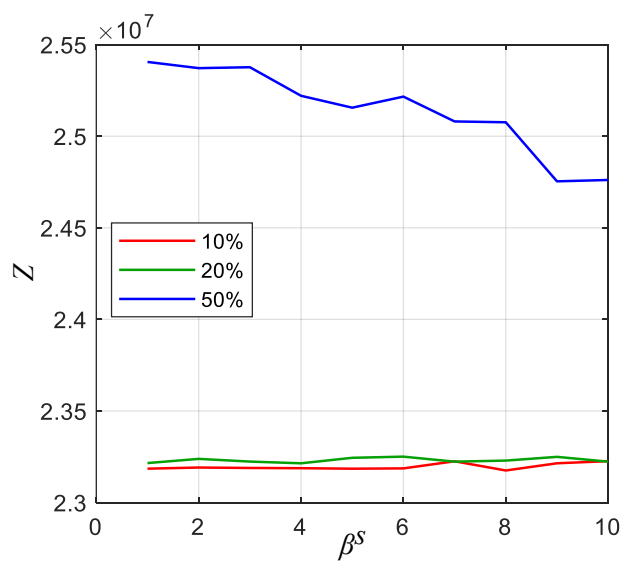

(a) Target value under various carpool cost and restricted proportion

$$
\psi=26 \%
$$

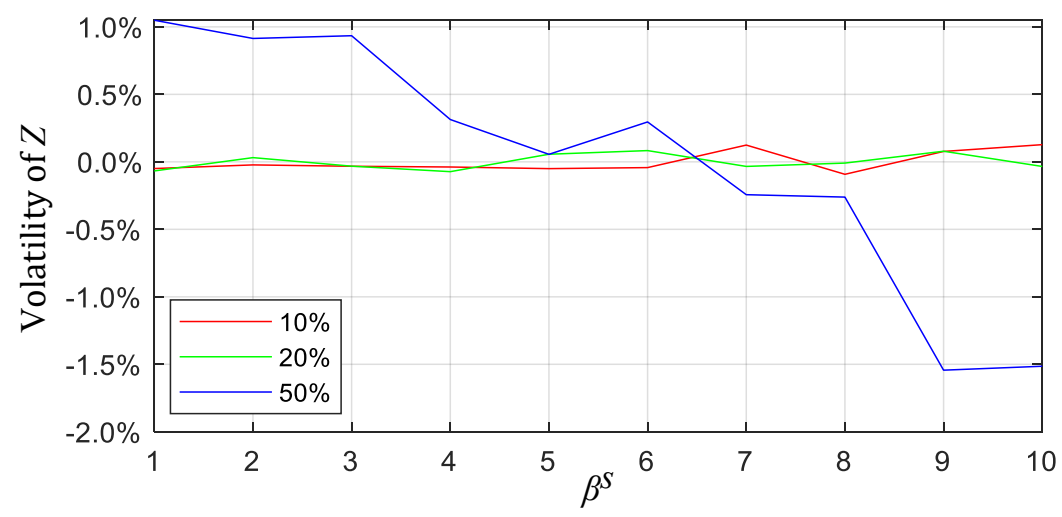

Figure 7. Sensitivity of target value to parameters.
(b)Target value under various carpool cost and exempted proportion $\lambda=50 \%$

From Figure 7a, there is a larger target value for larger restricted proportion, showing that the odd-and-even license plate rule performs best in this numerical experiment. When restricted proportion 
is set to $10 \%$ or $20 \%$, the target value is not sensitive to carpool cost and the maximum volatility of carpool cost is only $0.1 \%$. Whereas, when restricted proportion is set to $50 \%$, target value decreases with carpool cost and the maximum volatility is more than $1 \%$. These results indicate that the carpool exemption has little impact on the traffic equilibrium under TRP with small restricted proportion; consequently, the target value is slightly volatile with carpool cost. However, when restricted proportion reaches $50 \%$, the number of restricted private cars increases significantly and travel demand of restricted private cars that choose $\mathrm{CE}$ as alternative mode increases accordingly. At this time, the change of carpool cost will affect the mode choice behavior and traffic demand structure, further changing the network equilibrium. The subtle volatility indicates that there is no linear trend of target value under differentiated carpool cost. Consequently, it is necessary to determine the optimal exempted proportion combined with the actual carpool cost in practical application.

The sensitivity of target value to carpool cost and exempted proportion under the restricted proportion of $50 \%$ is further analyzed, as shown in Figure $7 \mathrm{~b}$. It can be seen that the target value increases with exempted proportion. However, there is an upper bound when exempted proportion is about $28-32 \%$, that is, increasing exempted proportion could not change the network equilibrium when it exceeds the bound. It indicates that the generated cost structure and given TRP scheme make about $30 \%$ private shift to $\mathrm{CE}$ at most. Furthermore, the upper bound of exempted proportion decreases with carpool cost and reaches its peak when carpool cost is $1 \mathrm{RMB} /$ trip. The results show that there is a larger adjustable space for exempted proportion when carpool cost is smaller.

\section{Conclusions}

To more effectively manage traffic demand and maintain the sustainability of transportation system, this paper focuses on the theoretical optimization of separate TRP and proposed TRP-CE. We propose an elastic demand model and corresponding equilibrium assignment model under TRP, based on which a bi-level programming model is developed for TRP optimization. Considering the negative impact of the mandatory TRP on the public acceptance, we propose a novel TRP with carpool exemptions, that is, a proportion of HOVs are allowed to travel in the restricted districts even if their license plate numbers are restricted. Then, the bi-level programming model is proposed for TRP-CE optimization by considering multi purposes of ensuring travel convenience, alleviating traffic congestion, and reducing the exhaust pollution, and GA algorithm is introduced to solve the optimal schemes. Finally, a numerical experiment is conducted to evaluate the effectiveness of proposed models.

In combination with comparative analysis between the theoretical optimal schemes of TRP and TRP-CE, main findings are concluded as follows. (1) When determining the restricted proportion, it is necessary to weigh in the feedback process of loss of travel convenience, improvement of traffic condition, and stimulation of travel intention. The proposed algorithm solving optimal TRP-CE scheme based on the implementation purpose can well meet the above requirements. (2) TRP-CE can not only improve the travel convenience, but also optimize the network demand structure and enhance the beneficial effect on alleviating exhaust pollution and traffic congestion. (3) The performance of an exempted proportion is not linearly related to the carpool cost with a given TRP scheme. Consequently, it is necessary to determine the optimal exempted proportion combined with the actual carpool cost in practical application.

This study provides a novel direction to make joint optimization of TRP and carpool strategy and contributes to the theoretical field of traffic demand management for sustainable transportation. Similar to HOV-lane management, a specific monitoring system is needed to monitor the occupancy of private cars, such as a monitoring system with infrared thermal imaging technology. Nevertheless, it is still easy to implement by adding the HOV monitoring function at license plate monitoring points, which are only necessary at the boundary of the restricted districts but not all internal roads.

To our knowledge, this paper is the first to propose TRP-CE from the concept to model to optimization algorithm. This study takes an initial step towards analyzing and understanding the 
effectiveness and advantages of TRP-CE. However, it is just the beginning and there exist some limitations worth further study. The present study does not consider enough factors that are crucial in terms of the success of CE combined with TRP (e.g., walking/cycling infrastructure, separated bus lanes, the accessibility of public transportation, etc.). Hence, a general direction to extend this study is to further explore the validity of TRP-CE by relaxing the assumptions and considering more influence factors, and try to identify and understand the societal, infrastructural, and operational characteristics that would affect the findings made here. In practice, TRP is usually supplemented with demand-management strategies such as improving service level of public transit, differentiated parking charges, increasing fuel surcharge and so on. However, analysis in this study does not involve aforementioned auxiliary strategies. Another possible direction for further research is to model the demand changing structure by considering these auxiliary strategies to make the model closer to reality. Furthermore, confronting the theoretical TRP-CE model with some other research, e.g., joining TRP with congestion charging or HOV/HOT lanes, would also be an important next step, one that could potentially generate more useful managerial insights for TDM.

Author Contributions: Conceptualization, X.W. and W.W.; formal analysis, X.W.; funding acquisition, W.W., D.Z. and X.H.; methodology, X.W.; project administration, W.W.; resources, D.Z.; software, W.Y.; supervision, W.W. and X.H.; validation, W.Y.; visualization, W.Y.; writing—original draft, X.W.; writing—review and editing, D.Z. and X.H. All authors have read and agreed to the published version of the manuscript.

Funding: This research was supported by the National Key R\&D Program of China (2019YFB1600200), National Natural Science Foundation of China (No. 51878166 and No.71801042), Scientific Research Foundation of Graduate School of Southeast University (No. YBJJ1837) and Jiangsu Graduate Research Innovation Program (No. KYCX18_0134).

Acknowledgments: The authors are grateful to reviewers for their constructive comments.

Conflicts of Interest: The authors declare no conflict of interest.

\section{References}

1. Schade, J.; Schlag, B. Acceptability of urban transport pricing strategies. Transp. Res. Part F-Traffic Psychol. Behav. 2003, 6, 45-61. [CrossRef]

2. Eriksson, L.; Garvill, J.; Nordlund, A.M. Acceptability of travel demand management measures: The importance of problem awareness, personal norm, freedom, and fairness. J. Environ. Psychol. 2006, 26, 15-26. [CrossRef]

3. Jia, N.; Zhang, Y.; He, Z.; Li, G. Commuters' acceptance of and behavior reactions to license plate restriction policy: A case study of Tianjin, China. Transp. Res. Part D-Transp. Environ. 2017, 52, 428-440. [CrossRef]

4. Lu, X. Effectiveness of government enforcement in driving restrictions: A case in Beijing, China. Environ. Econ. Policy Stud. 2016, 18, 63-92. [CrossRef]

5. Chen, L.; Li, G. Public acceptability and the impact factors of traffic restriction policy on tail number in Tianjin. J. Tianjin Univ. (Soc. Sci.) 2017, 19, 520-524.

6. Geng, J.; Long, R.; Chen, H.; Li, Q. Urban residents' response to and evaluation of low-carbon travel policies: Evidence from a survey of five eastern cities in China. J. Environ. Manag. 2018, 217, 47-55. [CrossRef]

7. Daganzo, C.F. A pareto optimum congestion reduction scheme. Transp. Res. Part B-Methodol. 1995, 29, 139-154. [CrossRef]

8. Nakamura, K.; Kockelman, K.M. Congestion pricing and roadspace rationing: An application to the San Francisco bay bridge corridor. Transp. Res. Part A-Policy Pract. 2002, 36, 403-417. [CrossRef]

9. Daganzo, C.F.; Garcia, R.C. A pareto improving strategy for the time-dependent morning commute problem. Transp. Sci. 2000, 34, 303-311. [CrossRef]

10. Shi, F.; Xu, G.; Liu, B.; Huang, H. Optimization method of alternate traffic restriction scheme based on elastic demand and mode choice behavior. Transp. Res. Part C-Emerg. Technol. 2014, 39, 36-52. [CrossRef]

11. Xu, G.; Shi, F.; Liu, B.; Huang, H. Model and algorithm of optimizing alternate traffic restriction scheme in urban traffic network. J. Cent. South Univ. 2014, 21, 4742-4752. [CrossRef]

12. Nie, Y. Why is license plate rationing not a good transport policy. Transportmetrica 2017, 13, 1-23. 
13. Ding, D. The optimal schemes for transportation network with carpooling based on speed limits and traffic restriction. Ph.D. Thesis, Southwest Jiaotong University, Chengdu, China, 2018.

14. Uncles, M.D. Discrete choice analysis: Theory and application to travel demand. J. Oper. Res. Soc. 1987, 38, 370-371.

15. de Cea Ch, J.; SotoO, A. A multi-modal supply-demand equilibrium model for predicting intercity freight flows. Transp. Res. Part B-Methodol. 2003, 37, 615-640.

16. Wei, X.; Wang, W.; Yu, W.; Hua, X.; Xiang, Y. A stochastic user equilibrium model under traffic rationing based on mode shifting rate. Sustainability 2020, 12, 5433. [CrossRef]

17. Nagurney, A.; Dong, J. A multiclass, multicriteria traffic network equilibrium model with elastic demand. Transp. Res. Part B-Methodol. 2002, 36, 445-469. [CrossRef]

18. Zhang, X.; Yang, H. The optimal cordon-based network congestion pricing problem. Transp. Res. Part B-Methodol. 2004, 38, 517-537. [CrossRef]

19. Yang, H.; Huang, H.-J. Carpooling and congestion pricing in a multilane highway with high-occupancy-vehicle lanes. Transp. Res. Part A-Policy Pract. 1999, 33, 139-155. [CrossRef]

20. Huang, H.-J.; Yang, H.; Bell, M.G.H. The models and economics of carpools. Ann. Reg. Sci. 2000, 34, 55-68. [CrossRef]

21. Qian, Z.S.; Zhang, H.M. Modeling multi-modal morning commute in a one-to-one corridor network. Transp. Res. Part C-Emerg. Technol. 2011, 19, 254-269. [CrossRef]

22. Song, Z.; Yin, Y.; Lawphongpanich, S. Optimal deployment of managed lanes in general networks. International. J. Sustain. Transp. 2015, 9, 431-441. [CrossRef]

23. Team, T.N.F.R. Transportation Networks for Research. Available online: https://github.com/bstabler/ TransportationNetworks (accessed on 28 June 2019).

24. Davis, L.W. The effect of driving restrictions on air quality in Mexico City. J. Political Econ. 2008, 116, 38-81. [CrossRef]

(C) 2020 by the authors. Licensee MDPI, Basel, Switzerland. This article is an open access article distributed under the terms and conditions of the Creative Commons Attribution (CC BY) license (http://creativecommons.org/licenses/by/4.0/). 